\title{
Erratum to: Analysis of umbu (Spondias tuberosa Arruda (Anacardiaceae)) in different landscape management regimes
}

\section{A process of incipient domestication?}

\author{
Ernani Machado de Freitas Lins Neto • Nivaldo Peroni • \\ Christine Maria Carneiro Maranhão • Maria Inês Sucupira Maciel • \\ Ulysses Paulino de Albuquerque
}

Published online: 20 September 2011

(C) Springer Science+Business Media B.V. 2011

Erratum to: Environ Monit Assess

DOI 10.1007/s10661-011-2280-7

The original version of this article unfortunately contained a mistake in Table 2 .

The corrected Table 2 is given below:

The online version of the original article can be found at http://dx.doi.org/10.1007/s10661-011-2280-7.

E. M. de Freitas Lins Neto $(\bowtie)$

Departamento de Biologia,

Universidade Federal do Piauí (UFPI),

Campus Professora Cinobelina Elvas, BR 135,

CEP 64900-000 Bom Jesus, Piauí, Brazil

e-mail: ernanilinsneto@ufpi.edu.br

\section{N. Peroni}

Departamento de Ecologia e Zoologia,

Universidade Federal de Santa Catarina,

Edifício Fritz Muller,

Florianópolis, Santa Catarina CEP 88040-970, Brazil

C. M. C. Maranhão

Departamento de Nutrição,

Universidade Federal de Pernambuco (UFPE),

Av. Prof. Nelson Chaves s/n, Cidade Universitária,

CEP 50670-901 Recife, Pernambuco, Brazil
M. I. S. Maciel

Departamento de Ciências Domésticas, Universidade Federal Rural de Pernambuco, Av. Dom Manoel de Medeiros s/n, Dois Irmãos, CEP 52171-900 Recife, Pernambuco, Brazil

U. P. de Albuquerque

Departamento de Biologia, Área de Botânica,

Universidade Federal Rural de Pernambuco,

Av. Dom Manoel de Medeiros s/n, Dois Irmãos, CEP 52171-900 Recife, Pernambuco, Brazil 


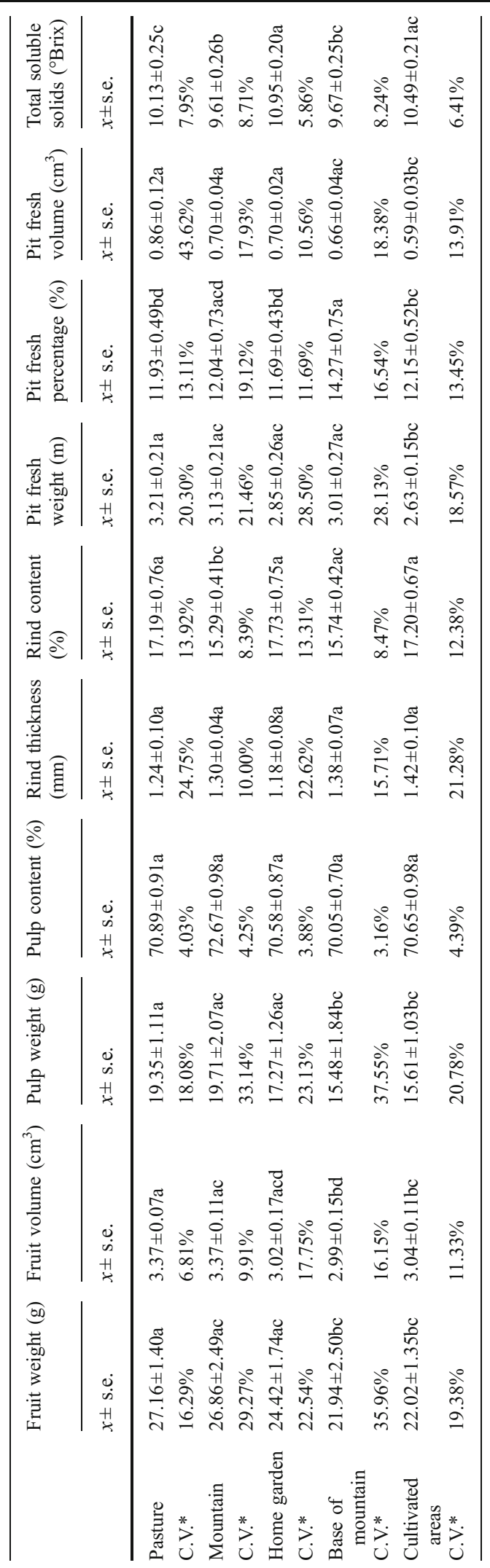

